DOSIER

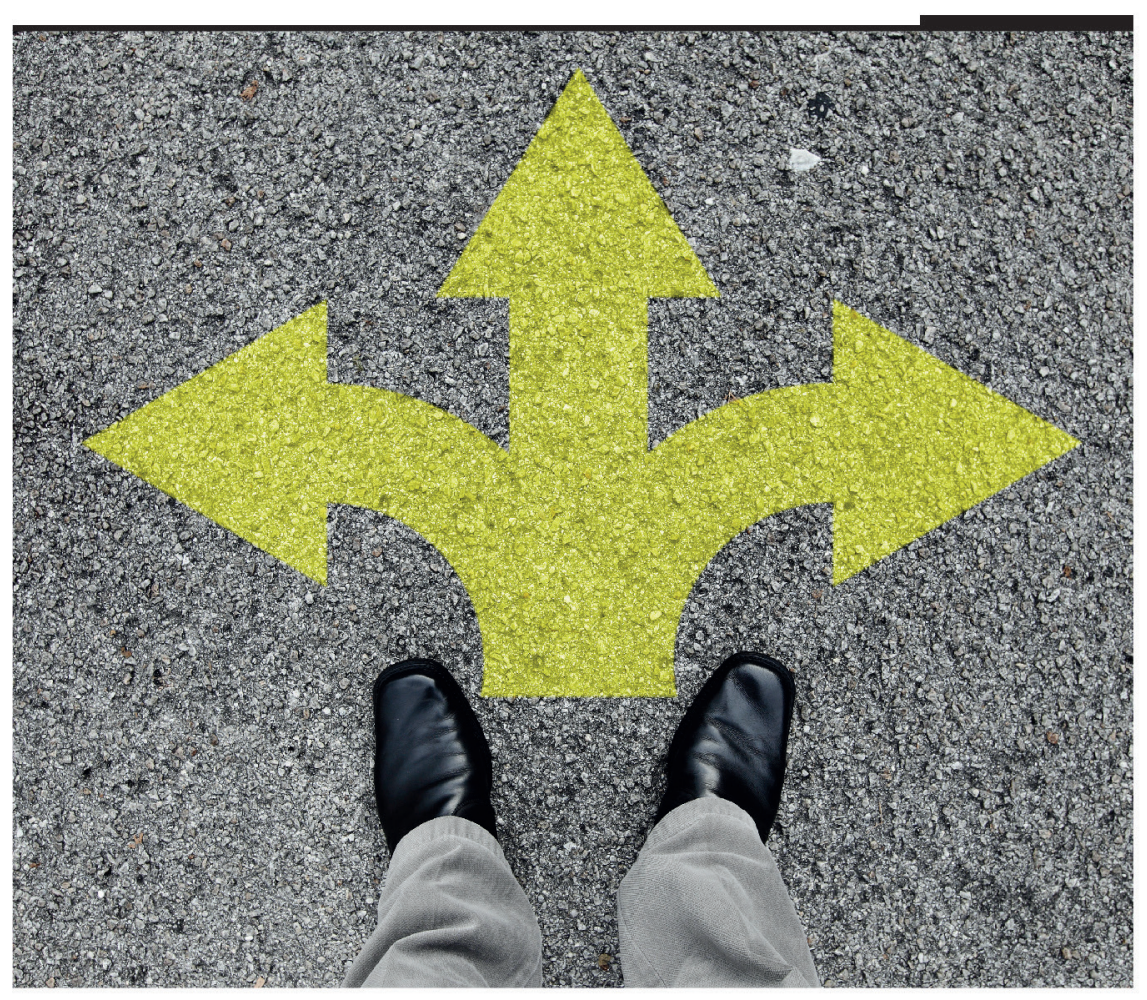





\title{
LA EDUCACIÓN MEDIÁTICA Y EL USO DE LOS RECURSOS TECNOLÓGICOS EN EL AULA EN EL CONTEXTO IBEROAMERICANO
}

\author{
MitTZy ARCINIEgA CÁCERES ${ }^{1}$ \\ Universitat Pompeu Fabra, España /mittzy.arciniega@upf.edu \\ MóNICA FIgUeRAS-MAZ² \\ Universitat Pompeu Fabra, España / monica.figueras@upf.edu
}

Recibido: 24/4/2019 / Aceptado: 22/7/2019

doi: 10.26439/contratexto2019.n032.4604

Resumen. El presente artículo tiene por objetivo presentar una reflexión en el contexto iberoamericano sobre el uso de los recursos digitales en el aula y su relación con la educación mediática para evidenciar puntos en común y estrategias diversas en el abordaje de ambos aspectos en los sistemas educativos, así como establecer los principales retos para una verdadera educación mediática en las aulas que aproveche las nuevas tecnologías. La metodología utilizada para dicho propósito se basa principalmente en una revisión sistematizada de la bibliografía sobre el tema entre los años 2012 y 2018. Además, el artículo aborda algunos resultados del proyecto "Competencias mediáticas de la ciudadanía en medios digitales emergentes en entornos universitarios" (20162019), del Plan Nacional de I+D+l del Gobierno español, e introduce las reflexiones al respecto de expertos en competencia mediática de la red interuniversitaria euroamericana Alfamed. Entre las principales conclusiones encontramos que existen iniciativas concretas y experiencias puntuales, pero no suponen un uso integral e innovador de las tecnologías de la información y de la comunicación. Más allá de la desigualdad en las oportunidades de acceso, los países iberoamericanos hacen un uso instrumental de los recursos tecnológicos en el aula y no se aprovecha su potencial para una verdadera educación mediática basada en la innovación en metodologías que pueda abordar los retos a los que se enfrenta el sistema educativo actual. El artículo, más que aportar

1 Doctora en Comunicación por la Universitat Pompeu Fabra, España. https://orcid.org/0000-0003-3720-048X 2 Doctora en Periodismo por la Universitat Autònoma de Barcelona, España. http://orcid.org/0000-0003-4912-4509 
nuevos resultados, supone una sistematización y un marco comparativo sobre la evolución, las experiencias innovadoras y la situación actual del uso de las tecnologías en la educación en distintos países iberoamericanos.

Palabras clave: educación mediática / dispositivos móviles / TIC en el aula / universidad / innovación

\title{
MEDIA EDUCATION AND USE OF TECHNOLOGICAL RESOURCES IN IBERO-AMERICAN CLASSROOMS
}

\begin{abstract}
The present article aims to present a reflection, within the Ibero-American context, on the use of digital resources in the classroom and its relationship with media education to highlight common points and diverse strategies in addressing both aspects in education systems, as well as to establish the main challenges for a real media education that could take advantage of new technologies in the classroom. The methodology used for this purpose is mainly based on a systematic review of the literature on the subject performed between the years 2012 and 2018. Moreover, the article addresses some of the results of the "Citizen's media competencies in emerging digital media in university environments" project (2016-2019) from the National Research, Development and Innovation ( $1+\mathrm{D}+\mathrm{I})$ Plan of the Spanish Government. Additionally, it presents reflections from experts in media competency of the Euro-American interuniversity research network Alfamed. The main conclusions include specific initiatives and particular experiences, but they do not imply an integral and innovative use of information and communication technologies. Beyond the inequality in access opportunities, Ibero-American countries make instrumental use of technological resources in the classroom and their potential is not used for a real media education based on innovation in methodologies that could address the challenges that current education system is facing. This article, more than providing new results, entails a systematization and a comparative framework on the evolution, innovative experiences and current situation of the use of technologies in education in different Ibero-American countries.
\end{abstract}

Keywords: Media education / mobile devices / ICT in the classroom / university / innovation 


\section{A EDUCAÇÃO MIDIÁTICA E O USO DE RECURSOS TECNOLÓGICOS EM SALA DE AULA NO CONTEXTO IBERO-AMERICANO}

Resumo. 0 presente artigo tem como objetivo apresentar uma reflexão no contexto ibero-americano sobre o uso de recursos digitais em sala de aula e sua relação com a educação midiática para destacar pontos em comum e diversas estratégias para abordar ambos os aspectos nos sistemas de ensino, bem como estabelecer os principais desafios para uma verdadeira educação em mídia nas salas de aula e que aproveitam as novas tecnologias. A metodologia utilizada para este fim baseia-se principalmente em uma revisão sistemática da literatura sobre o assunto entre 2012 e 2018. Além desta revisão da situação, o artigo está baseado em alguns dos resultados do projeto "Competências de mídia da cidadania em mídias digitais emergentes em ambientes universitários "(2016-2019) do Plano Nacional de P \& D do Governo Espanhol. Por fim, são apresentadas as reflexões, geradas a partir dos resultados obtidos neste projeto, de especialistas em competência midiática da rede interuniversitária euro-americana Alfamed. Entre as principais conclusões encontramos iniciativas específicas e experiências específicas, mas elas não implicam um uso integral e inovador das tecnologias de informação e comunicação. Além da desigualdade nas oportunidades de acesso, os países ibero-americanos fazem uso instrumental de recursos tecnológicos em sala de aula e seu potencial não é utilizado para uma verdadeira educação midiática baseada na inovação em metodologias que possam enfrentar os desafios aos que o atual sistema educacional está se enfrentando. 0 artigo, mais do que fornecer novos resultados, supõe uma sistematização e um quadro comparativo sobre evolução, experiências inovadoras e situação atual do uso de tecnologias em educação em diferentes países ibero-americanos.

Palavras chave: Alfabetização midiática / dispositivos móveis / TIC na sala de aula / universidade / inovação 


\section{INTRODUCCIÓN}

La comunicación mediática es el resultado de una serie de interacciones entre personas y tecnologías en un contexto social, económico y cultural determinado. Las personas crean mensajes que reciben otras personas, y lo hacen a través de unas tecnologías usando unos códigos compartidos en contextos determinados. En la actualidad, y gracias a las nuevas tecnologías, las personas que acceden a ellas están en disposición no solo de consumir sino también de producir mensajes, de manera que es necesario que la ciudadanía sea competente en el ámbito mediático; el fenómeno actual de las fake news es un buen ejemplo de esta necesidad. Ahora bien, diversos estudios muestran carencias significativas en la formación mediática de la ciudadanía española (Ferrés, Aguaded-Gómez y García-Matilla, 2012) y es por ello que surgió el proyecto "La enseñanza universitaria ante la competencia mediática en un entorno digital", llevado a cabo entre el 2011 y el 2014 y financiado por el Ministerio de Economía y Competitividad dentro del plan estatal español de l+D+l. El objetivo fue analizar la formación en educación mediática que ofrecían las universidades españolas en los estudios de comunicación y educación por ser estos los que más trascendencia tienen después en la formación de la ciudadanía. Los resultados de esta investigación evidenciaron también graves carencias en la formación en educación mediática ofrecida en el contexto universitario (Tucho, Fernández-Planells, Lozano y Figueras-Maz, 2015; López y Aguaded, 2015; Ferrés y Masanet, 2015; Masanet, Contreras y Ferrés, 2013; Marta-Lazo, Grandío y Gabelas, 2014).

El presente artículo se enmarca en un nuevo proyecto titulado "Competencias mediáticas de la ciudadanía en medios digitales emergentes en entornos universitarios" (2016-2019) del Plan Nacional de I+D+l del Ministerio de Industria, Economía y Competitividad de España. El proyecto tiene por objetivo general analizar la situación de uso y consumo de medios digitales (teléfonos inteligentes y dispositivos móviles) y sus potencialidades para dinamizar la apropiación activa, crítica e inteligente de los ciudadanos en contextos democráticos de la sociedad digital. Específicamente, el proyecto pretende diagnosticar carencias, necesidades y prácticas innovadoras en la universidad para el fomento de la competencia mediática en medios emergentes digitales interactivos y potenciar estrategias educomunicativas en este nivel superior de enseñanza.

El debate sobre la introducción de las tecnologías de la información en el aula viene de lejos, desde la aparición y generalización de los equipos informáticos en la sociedad. No obstante, con la emergencia de los dispositivos móviles (teléfonos inteligentes

y tabletas) ha resurgido. Pese a no estar resuelto el primer debate, debido en buena parte a las dificultades de acceso aún en muchos lugares, la aparición masiva de estos nuevos dispositivos obliga a plantearlo de nuevo y en otros términos. Tal como pone de manifiesto el documento "Enfoques estratégicos sobre las TIC en educación en América Latina y el Caribe" (Unesco, 2014), el sistema educativo de hoy requiere una reconversión 
estructural de los procesos y las prácticas de aprendizaje, con la consecuente actualización de contenidos, que se adapten a las nuevas formas de ser y aprender, ya no solo enfocados en los cambios tecnológicos que han supuesto las herramientas digitales, sino orientados a una reforma integral de la manera como los niños, las niñas y los jóvenes perciben el mundo, recepcionan, producen y gestionan la información y el conocimiento, y el rol que la educación debe tener. El mismo informe señala, además, que la introducción de las TIC en el aula implica, primeramente, un desafío pedagógico, ya que la literatura existente evidencia que, hasta el momento, las experiencias en América Latina y el Caribe no han sido del todo efectivas en relación con la calidad de educación. Los datos plasmados en este informe de la Unesco (2014) coinciden con los obtenidos en el contexto español en la investigación "Competencias mediáticas de la ciudadanía en medios digitales emergentes en entornos universitarios", antes citada, en que se enmarca el presente artículo.

Así, según se desprende de ambos documentos, el modelo sigue una lógica de importación, es decir, basado en la incorporación de dispositivos y softwares en el aula, sin tener claros los objetivos pedagógicos ni, mucho menos, la manera de alcanzarlos. Como consecuencia, muchas veces las tecnologías, lejos de ser protagonistas del proceso de aprendizaje, han pasado a tener un rol secundario en un contexto de prácticas educativas tradicionales. De esta manera se ha focalizado el uso de las nuevas tecnologías en el ámbito tecnológico, lo que ha supuesto, por un lado, la consecuente pérdida de potencial que podrían ofrecer estas herramientas y, por otro, en algunos casos, las dificultades y "analfabetismo tecnológico" de los docentes, que se ven superados por sus estudiantes, los que Prensky (2001) definió como nativos digitales.

Según este autor, los nativos digitales son menores de 30 años, por tanto, han nacido y crecido con la tecnología a su alcance y eso significa que se desenvuelven de forma natural y casi innata en este entorno digital. Sin embargo, esta destreza tecnológica no garantiza automáticamente que sean personas competentes en el uso de las TIC. En el mejor de los casos podríamos decir que lo son en una de las dimensiones de la competencia mediática, el uso instrumental de las tecnologías. Los jóvenes suelen obtener puntuaciones muy bajas en todas las dimensiones de la educación mediática excepto en la tecnológica, lo que evidencia la necesidad de una educación mediática integral (Masanet, Contreras y Ferrés, 2013). Así, en España, los jóvenes disponen de habilidades técnicas y de producción, pero carecen de otras igualmente importantes, como las estéticas o la actitud crítica frente a los medios, según la clasificación de dimensiones de Ferrés y Piscitelli (2012).

El presente artículo tiene por objetivo una reflexión en el contexto iberoamericano sobre el uso de los recursos digitales en el aula y su relación con la educación mediática para evidenciar puntos en común y estrategias diversas en el abordaje de ambos 
aspectos en los sistemas educativos, así como establecer los principales retos para una verdadera educación mediática en las aulas que aproveche las nuevas tecnologías.

\section{METODOLOGÍA}

Los datos utilizados para el presente artículo se han obtenido a partir de la revisión de literatura científica; los resultados de la investigación "Competencias mediáticas de la ciudadanía en medios digitales emergentes en entornos universitarios" (2016-2019) del Plan Nacional de I+D+I del Ministerio de Industria, Economía y Competitividad de España, en concreto, los grupos de discusión realizados con estudiantes y profesores, así como los cuestionarios aplicados a responsables de innovación docente y a expertos euroamericanos de la red interuniversitaria Alfamed.

En cuanto a la revisión de literatura, esta se ha llevado a cabo como método exploratorio que nos ha permitido identificar las investigaciones más relevantes en relación con el tema de estudio a partir del año 2012. Para ello, se han utilizado bases de datos de Scopus, Web of Science y Google Scholar a partir del criterio de búsqueda definido por las palabras claves (educación mediática, dispositivos móviles, TIC en el aula, universidad, innovación) y por filtros, como fecha de publicación, lengua (inglés, español y catalán) y calidad (priorización de publicaciones indexadas).

Respecto a los grupos de discusión, se realizaron cuatro con estudiantes universitarios de 19 a 23 años de las ciudades de Barcelona, Madrid, Sevilla y Huelva en el periodo mayo-junio del 2018.

Finalmente, se incluyen los resultados parciales del cuestionario de cuarenta y cinco preguntas aplicado a ocho expertos euroamericanos de la red interuniversitaria Alfamed (https://www.redalfamed.org/) con el objetivo de conocer sus percepciones, preocupaciones, motivaciones y retos.

\section{LA EDUCACIÓN EN MEDIOS VERSUS EDUCACIÓN CON MEDIOS}

Existe una extensa literatura científica sobre el concepto de educación mediática o términos similares como "educación en medios", "educación en comunicación audiovisual", o bien, "alfabetización mediática", "alfabetización informacional”, "alfabetización audiovisual". Se habla también de "alfabetización en tecnologías de la información y de la comunicación (TIC)" o "alfabetización digital". Tanto la Unesco como la Alianza de Civilizaciones hablan de "alfabetización mediática e informacional" y la Comisión Europea utiliza el concepto media literacy.

Podríamos decir que lo informacional se refiere al acceso, tratamiento y conversión en conocimiento de la información mientras que lo audiovisual pone más el acento 
en los medios audiovisuales y, finalmente, lo digital hace referencia a la capacidad de búsqueda, procesamiento, comunicación, creación y difusión de informaciones a través de las tecnologías. En algunos casos, digital se ha referido solo a la introducción de tecnologías en la educación, es decir, a la educación "con" tecnologías y no "en" tecnologías. Lo mediático, en cambio, se usa como integración de estos tres conceptos y entendemos que incluye tanto los medios de comunicación tradicionales (prensa, radio, televisión) como los nuevos medios (ordenadores y dispositivos digitales con acceso a internet). En muchos casos también desde esta premisa se ha confundido la educación en medios con la educación con medios. En los últimos años ha aparecido un nuevo término, "alfabetización o educación transmedia" (Frau-Meigs, 2012; Scolari, 2016). Estos autores proponen usar "transmedia" en lugar de "mediática" ante la realidad actual del ecosistema mediático y del contexto de cultura participativa.

En cualquier caso, lo importante es el contenido que reflejan estos conceptos. La educación mediática, o como se defina, debe referirse al conjunto interrelacionado y complejo de conocimientos, destrezas y actitudes que permitan a la ciudadanía desenvolverse eficazmente en el contexto mediático actual y adaptarse adecuadamente a los cambios rápidos y a entornos distintos. En este sentido, defendemos la propuesta de dimensiones sobre competencia mediática desarrollada por Ferrés y Piscitelli (2012), que nació de las observaciones y sugerencias de más de cuarenta expertos en educación mediática del ámbito iberoamericano. Esta propuesta estructura la educación mediática en seis dimensiones básicas: lenguajes, tecnología, ideología y valores, procesos de interacción, procesos de producción y difusión, y estética. En definitiva, la dimensión tecnológica de la competencia mediática es solo una de la seis que entendemos que comprende el concepto. Otra cosa distinta es que se aprovechen las tecnologías para la educación mediática en todas sus dimensiones.

\section{LOS NUEVOS ROLES EN EL PROCESO EDUCATIVO}

Según el documento "Enfoques estratégicos sobre las TIC en educación en América Latina y el Caribe" (Unesco, 2014), la Unesco tiene un claro encargo de los estados miembros para "abordar los temas clave, las tensiones y las posibilidades al alcance de las políticas públicas que permitan aprovechar el potencial de las TIC a favor de la educación y el desarrollo" (Unesco, 2014, p. 13) y es justamente aquí donde la educación mediática podría ser una de las bases.

En este mismo documento, la Oficina Regional de Educación de la Unesco para América Latina y el Caribe -OREALC/Unesco Santiago- señala que existen dos ejes de desarrollo prioritario en el camino para que estas tecnologías realmente aporten una mejora sistémica al sistema educativo, sobre todo en relación con la inclusión y el acceso igualitario (Unesco, 2014). Como primer eje hace hincapié en las nuevas prácticas 
educativas y, como segundo eje, en la medición de aprendizajes. Y es justamente en relación con las nuevas prácticas que hacemos alusión a la educación mediática.

La finalidad es potenciar el desarrollo de prácticas de reciprocidad, cooperación y participación, lo que significa incentivar el aprendizaje activo y, al mismo tiempo, respetar la diversidad de formas de ser y aprender. Sin embargo, tanto en Latinoamérica como en España se evidencian importantes críticas, tanto de estudiantes como del profesorado, respecto al diseño pedagógico actual y al planteamiento de contenidos, puesto que no responden a las necesidades de la sociedad actual en la que los roles se intercambian y las maneras de enseñar y aprender se actualizan constantemente (Cuban, 2003; Lugo, 2010 y Andrada, 2018).

Así, se pone sobre la mesa la necesidad de redefinir los roles de alumnado y profesorado. Las TIC dotan a los estudiantes de mayor autonomía y responsabilidad en el proceso de aprendizaje, lo que obliga al profesorado a salir de su rol como único e incuestionable proveedor de conocimiento; es el fin de una transmisión lineal y jerárquica del saber. Tal como afirma Lugo (2010), esto genera una serie de sentimientos en los docentes, desde incertidumbres, hasta temor y presión por responder a las expectativas del alumnado, lo que obliga a repensar y reinventar creativamente el rol del docente como facilitador. El aprendizaje deja de ser unidireccional para adquirir una multidireccionalidad, el saber deja de estar en unos pocos y se descentraliza en muchos. Hoy, estamos ante un fenómeno educativo que trasciende el saber individual. Según Pierre Lévy (2004), en una sociedad en red nadie posee el saber absoluto, sino que todas las personas saben algo y construyen el conocimiento de manera colectiva en tanto que todo ese saber es compartido y alimentado por la propia comunidad.

De esta manera es necesario abordar los recursos digitales más allá del ámbito tecnológico y entenderlos como nuevos escenarios, formas de expresión, socialización y acción. Ello significa entender las nuevas narrativas, las diferentes formas de construir la identidad y percibir el mundo desde las mediaciones. Pero este entendimiento no es posible sin un trabajo de educación mediática que permita tender puentes entre unos modos de enseñar y aprender homogéneos a un nuevo modelo de aprendizaje colectivo basado en la diversidad y con especial foco en lo personal y lo social.

\section{LA POCA IMPLEMENTACIÓN EN IBEROAMÉRICA}

Tal como se reflexiona en el informe "Enfoques estratégicos sobre las TIC en educación en América Latina y el Caribe" (Unesco, 2014), después de más de veinte años centrando los esfuerzos en Latinoamérica en habilitar las escuelas tecnológicamente, con equipamientos y posibilidades de conexión, y una gran inversión económica, la decepcionante realidad es que menos de dos de cada diez docentes afirman que utilizan con frecuencia 
los ordenadores en el aula; solo tres o cuatro los usan ocasionalmente y el resto, cuatro o cinco de cada diez, declaran que no los emplean nunca para enseñar.

Sin embargo, la decepción va más allá. Cuando a los docentes que afirman que utilizan las TIC se les pregunta sobre el uso que les dan, en muchos casos responden que aprovechan los ordenadores para procesar textos $u$ otras aplicaciones básicas sin explotar su potencial para transformar las formas tradicionales de enseñanza. Así, a pesar de todos los recursos económicos destinados, parece que estos no han dado los frutos esperados (Cuban, 2003).

Los dispositivos móviles, como los teléfonos inteligentes, no escapan de esta situación. Pese a ser más atractivos e integrados a la vida de personas adultas, por tanto, de muchos profesores, su uso en el aula sigue siendo puramente instrumental. Un informe que la Organización para la Cooperación y Desarrollo Económicos (OCDE) publicó en el 2015, Students, Computers and Learning: Making the Connection, PISA, determina que, a pesar de lo esperado, la tecnología móvil no ha contribuido de manera significativa a reducir la brecha existente al día de hoy, lo que supone una afirmación poco alentadora. "Si los estudiantes utilizan un teléfono inteligente para copiar y pegar información, es muy difícil que puedan desarrollar habilidades de pensamiento crítico" (OCDE, 2015, p. 3), cita el informe.

Aunque parezca paradójico, dado que lo digital es el entorno natural de muchas personas jóvenes, investigaciones como la que enmarca este artículo ponen de manifiesto una cierta negatividad por parte de los estudiantes de universidades españolas con respecto a la "escolarización de la tecnología" tal como se plantea en la actualidad. Así lo manifestaron algunos de los estudiantes de segundo curso en adelante (de 19 a 23 años) participantes en los cuatro grupos de discusión que se realizaron en Barcelona, Madrid, Sevilla y Huelva en el periodo mayo-junio del 2018: “En una clase nos hacían ver un video y comentar por WhatsApp y nadie respondía. Los estudiantes responden 'qué bien' o con emoticones" (Estudiante mujer, Publicidad y Relaciones Públicas, Universitat Ramon Llull, 2. ${ }^{\circ}$, Barcelona, grupo de discusión realizado el 3 de mayo del 2018).

\footnotetext{
Hubo un intento de hacer una clase por Twitter en la asignatura de Sociología. Usaba hashtag. Al principio eran varios conectados, luego fue fracasando. Se te olvida y eran temas aburridos como democratización del consumo. Todos los martes de 6 a 8. Al principio éramos 30, pero al final se te olvidaba la hora. (Estudiante hombre, Publicidad y Relaciones Públicas, Universitat Pompeu Fabra, 4.․, Barcelona, grupo de discusión realizado el 3 de mayo del 2018)
}

En la publicación "Experiencias evaluativas de tecnologías digitales en la educación”, de Fundación Telefónica y Unesco (Fundación Telefónica Vivo, 2016), se pone de manifiesto que la población en general, y la joven en particular, tiene una alta predisposición al uso de las TIC en la esfera personal. Sin embargo, ello no siempre significa 
que las personas jóvenes estén totalmente de acuerdo con su uso en entornos educativos. Cuestionan, sobre todo, el uso de estas herramientas en entornos tradicionales, en donde, lejos de sacarles provecho, las TIC se convierten en una extensión de la metodología clásica.

Durante las últimas décadas se han realizado esfuerzos por parte de España y muchos países de Latinoamérica para incorporar las TIC a los procesos educativos y, en casos puntuales, incorporar la perspectiva de la educación mediática. Estas iniciativas han mostrado importantes logros en relación con la reducción de la brecha digital, pero en la mayoría de los casos los resultados actuales distan mucho de los imaginados hace veinte años, cuando las TIC irrumpieron en el aula.

Aunque la gran mayoría de retos son compartidos en toda lberoamérica, el IV Estudio sobre el Uso de la Tecnología en la Educación (Blïnk Learning, 2018) señala que en el caso de Latinoamérica, especialmente Perú y Chile, los docentes manifiestan la necesidad de dotar de un sentido inclusivo a las tecnologías de la información y de la comunicación con iguales oportunidades de acceso, de lo contrario, la presencia de las TIC como un elemento de élite no hará más que ampliar la brecha entre los más y los menos favorecidos. Obviamente, la principal distancia para adquirir la competencia mediática es la que existe entre los que pueden acceder a las tecnologías y los que no.

Esta situación se corrobora si hacemos un breve repaso por algunas de las iniciativas desarrolladas en las últimas décadas en el contexto latinoamericano, por ejemplo las de los años noventa en Costa Rica y Chile, a través del Plan de Informática Educativa, y más recientemente, el Plan Ceibal en Uruguay, el programa Conectar Igualdad en Argentina, el proyecto Una Laptop por Niño de Perú, la iniciativa Colombia Aprende y el programa Habilidades Digitales para Todos del Gobierno de México, entre otras.

Resulta interesante destacar el caso de Brasil, en que las iniciativas de educación mediática están casi siempre lideradas por entidades de educación no formal, tal como afirman Pegurer y Martínez-Cerdá (2016). Los proyectos llevados a cabo con jóvenes desde las ONG y entidades de educación no formal parecen cubrir los vacíos existentes en las actuales políticas públicas orientadas a la educación mediática. Para los autores, la situación ideal para el país sería unificar y complementar iniciativas de las políticas públicas con las de educación no formal, todo ello considerando las grandes diferencias sociales existentes en el país.

En el caso de Chile, aunque se enmarque fuera de las aulas, podemos destacar el rol que jugaron las TIC en las movilizaciones estudiantiles del 2006, en la llamada revolución de los pingüinos, lo que permite entender el uso que brindan los jóvenes chilenos a las nuevas formas de comunicación. En la misma línea de la participación ciudadana y la educación mediática, Argentina está orientada a fortalecer el sentido democrático de los jóvenes. Este país, además, se focaliza en la generación de debates y análisis de 
los medios, que han estado fuera de las escuelas durante muchos años. Los docentes argentinos que formaron parte de la resistencia en 1984, cuando la democracia volvió al país, son ahora docentes abiertos y preparados para incorporar la educación mediática en sus aulas (Unesco, 2014).

Colombia es quizás la región de Latinoamérica que más ha centrado sus esfuerzos en la educación mediática, más allá de alfabetización TIC. Muchos de los proyectos nacionales en esta materia incluyen dentro de sus objetivos o metodología la reflexión crítica de contenidos de medios de comunicación, como por ejemplo el trabajo de Barón, Acevedo y Luque (2002), quienes desarrollan la investigación "Internet, guerra y paz en Colombia", centrada en los nuevos medios de comunicación y su incidencia en la cultura de niñez. Este trabajo tiene como antecedente directo, en el año 1998, la Dirección de Desarrollo Educativo de la Secretaría de Educación y Cultura de Antioquia (Seduca), que diseña una encuesta para determinar la pertinencia del medio como herramienta pedagógica en el aula (Martín-Barbero y Téllez, 2006). En este contexto se encuentra el proyecto Mirando cómo Miramos, desarrollado por Sandoval-Romero y Arenas (2010), que propone un modelo de formación en recepción crítica para diferentes públicos. Finalmente, desde una propuesta de intervención en investigación en Colombia, Vega y Lafaurie (2013) presentan los resultados del proyecto desarrollado en el año 2008 con niños de 7 a 11 años, en el que incorporan un taller de educación mediática.

\section{LA OPINIÓN DE INVESTIGADORES DE LA RED ALFAMED}

La mayoría de expertos de la red Alfamed considera que los dispositivos móviles son más bien elementos de dispersión, distracción y ansiedad y declaran que no utilizan las TIC para trabajar el consumo crítico de los estudiantes y mucho menos como productores responsables, éticos y creativos. Son críticos con el empleo por los estudiantes de los dispositivos móviles, sin embargo, parecen no considerar que sea parte de su función potenciar un uso integral que explote sus posibilidades.

Con el uso de dispositivos móviles, [los estudiantes] se ubican con respecto al tema, pero no profundizan en la misma [sic]. Se quedan con lo básico o primero que consultan. (Experto y docente UniMinuto, Colombia, respuesta del cuestionario online, 19 de noviembre del 2018)

En términos generales, los profesores son conscientes de la necesidad de prepararse con respecto al uso de las nuevas tecnologías en el aula y específicamente sobre los dispositivos móviles, sin embargo, al ser preguntados sobre el tipo de formación que desean recibir la gran mayoría afirma que requiere formación técnica y raramente se hace mención al aprendizaje de un nuevo enfoque pedagógico motivado por las TIC. A continuación, ilustramos una de las pocas declaraciones que hace alusión a los procesos pedagógicos: 
La medida más urgente es la implementación de procesos pedagógicos y didácticos, como una alfabetización en lo digital, pues conozco muchos compañeros que no lo hacen por desconocimiento o también porque no les gusta. (Docente e investigador, Universidad Católica Luis Amigó, Medellín, Colombia, respuesta del cuestionario online, 19 de noviembre del 2018)

Aunque reconocen la necesidad de formación, sobre todo desde el punto de vista técnico, al preguntárseles si precisan ayuda de sus estudiantes para utilizar los dispositivos móviles en las actividades de sus asignaturas, la respuesta es rotundamente negativa, lo que evidencia la dificultad actual para asumir un cambio en los roles de docentes y estudiantes. Ello va en la línea del uso que actualmente realizan de estos dispositivos en clase, puesto que manifiestan, en términos generales, que aplican una metodología de trabajo individual, contraria al aprendizaje colectivo promovido por las TIC. Por otra parte, se evidencia un uso superficial y utilitario de los recursos tecnológicos en clase, como, por ejemplo, redactar y editar textos y gráficos.

Respecto a las ventajas de utilizar dispositivos móviles en el aula, se hace alusión mayoritariamente a la diversidad de fuentes, la inmediatez, el acceso a la información, entre otros, pero se mencionan poco las posibilidades de trabajo colectivo, participación democrática, intercambio de roles alumno/docente o la posibilidad de adaptar contenidos y personalizar la educación. Sin embargo, cabe destacar algunas opiniones que sí tienen una visión integral e innovadora:

...el uso del dispositivo no puede ser para fines mecánicos y cumplir con el uso, sino para lograr un mejor aprendizaje. (Experta del Área Sociohumanística, Universidad Técnica Particular de Loja, respuesta del cuestionario online, 22 de junio del 2018)

El trabajo con dispositivos móviles debe ser útil para el proceso de enseñanzaaprendizaje; que resulte interesante para los estudiantes; que colaboren con el desarrollo de nuevas competencias. (Investigador Universidad de Salamanca, respuesta del cuestionario online, 22 de junio del 2018)

La enseñanza debe ser interactiva y colaborativa y que permita interaccionar entre estudiantes, profesorado y profesionales externos/as. (Investigador Universidad de Oviedo, respuesta del cuestionario online, 22 de junio del 2018)

Debe permitir que cada uno avance a su ritmo y solucione las necesidades que se le presenten. (Investigador Universitat Politècnica de Catalunya, respuesta del cuestionario online, 24 de enero del 2019)

Como pasa entre los conceptos de educación con medios y en medios, es importante no confundir el concepto de innovación a través de la tecnología con el de la introducción de la tecnología en el aula y que, per se, sea innovador. Como señalan Fueyo, RodríguezHoyos y Linares, "la transformación de las prácticas pedagógicas es una tarea que no se puede reducir de forma exclusiva a la utilización de dispositivos tecnológicos en las 
aulas, sino que ha de suponer una modificación que ha de afectar, necesariamente, a otros elementos curriculares: la selección de contenidos, la evaluación, las estrategias metodológicas empleadas, etcétera" (2015, p. 33).

\section{ESTRATEGIAS UTILIZADAS Y RETOS EN EL USO DE LAS TIC}

Introducir tecnologías en el aula no supone automáticamente una mejora en la calidad de la enseñanza ni en los resultados de aprendizaje. Más aún, no es necesaria la tecnología para conseguir esa mejora. Así, según el informe español OCDE 2012, en su volumen I sobre resultados y contexto, se evidencia que los países con mejores resultados tienen perfiles muy variados e incluso opuestos. Por ejemplo, Finlandia, Corea del Sur y Japón, países donde el uso de la tecnología en las aulas es bajo, obtienen muy buenos resultados educativos; sin embargo, resultados igual de buenos son observados en Singapur, Países Bajos o Estonia, donde el uso de la tecnología en los centros educativos es elevado (OCDE, 2015).

En la misma línea, el "Estudio sobre la inclusión de las TIC en los centros educativos en aulas Fundación Telefónica" (Organización de Estados Americanos y Fundación Telefónica, 2018), señala que Singapur, por ejemplo, está cambiando el enfoque aplicado en su sistema educativo por un sistema más flexible "basado en habilidades", otorgando especial importancia al trabajo por proyectos con la tecnología en el aula como protagonista del cambio. Otro ejemplo lo podemos encontrar en el modelo de la Escuela Nueva de Colombia, basado en la solución de problemas, que permite que participen cinco millones de alumnos de dieciséis países. En ambos casos, tanto en Singapur como en Colombia, estos cambios estructurales del modelo educativo han requerido modificar los procesos y las prácticas pedagógicas, pero, sobre todo, ha sido indispensable un cambio en las maneras de hacer de los docentes con respecto al alumnado.

En algunos países de Latinoamérica la estrategia de promoción de la educación mediática ha sido selectiva, es decir, descentralizar los esfuerzos a dimensiones o ámbitos concretos y no a todos los que confiere la competencia mediática. Por ejemplo, según Pegurer y Martínez-Cerdá (2016), en Brasil la educación mediática se ha focalizado principalmente en la producción de contenido mientras que, según Morduchowicz (2017), en Argentina se ha centrado más en la participación democrática y la libertad de expresión. Sin embargo, de acuerdo con ambos trabajos, se evidencia en los dos países la necesidad de promover y fortalecer una cultura política democrática en los jóvenes, de manera que entiendan los principios esenciales de la democracia. Tanto el trabajo de Pegurer y Martínez-Cerdá (2016) como el de Morduchowicz (2017) ponen de manifiesto que, en ambos países, los estudiantes necesitan aprender y valorar el derecho a expresarse libremente y el libre derecho al acceso de información verídica, lo que implica contar con destrezas suficientes para contrastar la veracidad de las fuentes. Además, 
estas investigaciones hacen énfasis en la importancia de explorar las posibilidades de las TIC más allá del ocio y, por otra parte, hacer un uso crítico y responsable de ellas.

En el caso de México, según Hernández y Bautista (2017), las políticas sobre el uso de la tecnología educativa no están bien definidas y son realmente los organismos independientes los que están desarrollando un rol vital en la divulgación sobre investigación del uso de las TIC en los ámbitos nacional e internacional. Estos autores hacen hincapié en la necesidad de formar a los docentes en materia de educación y TIC.

En esta misma línea, el IV Estudio sobre el Uso de la Tecnología en la Educación (Blïnk Learning, 2018) indica que la estrategia principal tanto en México como en España ha sido centrar la atención en la formación docente. La formación del profesorado en todos los niveles educativos y, especialmente, de los futuros docentes en educación, desde preescolar hasta la universidad, es clave para el futuro de la competencia mediática entre la ciudadanía. Hoy la comunicación es un componente clave en los procesos de socialización y de aprendizaje. Por tanto, la formación universitaria en las titulaciones de educación es una estrategia prioritaria, especialmente en educación infantil (Andrada, 2018).

Según el IV Estudio sobre el Uso de la Tecnología en la Educación (Blïnk Learning, 2018), España hace especial hincapié en la necesidad de motivar a los estudiantes. Lo que sucede hoy es que los estudiantes manifiestan que el uso que se da a las TIC en el aula no genera un impacto real, que su utilización es irrelevante y que, sin embargo, sí supone un esfuerzo extra ante el cual no encuentran la recompensa. En este mismo sentido coinciden los resultados obtenidos en los grupos de discusión con estudiantes universitarios españoles en el proyecto "Competencias mediáticas de la ciudadanía en medios digitales emergentes en entornos universitarios":

No se puede transmitir lo que no se siente. Hay una viñeta diciendo soy innovador y utiliza el libro digital y la pizarra, pero igual que tradicionalmente. Cambia el soporte, pero no la metodología, no las técnicas. (Estudiante mujer, educación primaria, 3., Universidad de Huelva, grupo de discusión realizado el 5 de junio del 2018)

Forzar a un profesor a hacer una cosa que domina, que no le agrada termina desmotivándonos. Nos dicen que nos animemos, que "qué nos pasa". Nosotros valoramos que hagas eso, pero hay otras maneras. No sé si se les obliga desde la facultad o es para tener un mejor prestigio. Pareciera que los profesores han de ser modernos. Cuando intentan usar la tecnología y no les funciona, el profesor pierde el control. Cada profesor debe sentirse cómodo en lo que se hace. (Estudiante hombre, Publicidad y Relaciones Públicas, Universitat Pompeu Fabra, 3. ${ }^{\circ}$, Barcelona, grupo de discusión realizado el 3 de mayo del 2018)

Si el profesor quiere, que use la tecnología, pero que no se siente forzado. No se debe sentir presionado porque todo el mundo lo hace porque a lo mejor su método es igual de bueno o mejor solo hablando que otro que tiene mil videos, mil PowerPoint e historias. (Estudiante mujer, Publicidad y Relaciones Públicas, 
Universitat Ramon Llull, 2. ${ }^{\circ}$, Barcelona, grupo de discusión realizado el 3 de mayo del 2018)

Pese a ciertos matices en uno u otro país, la tendencia generalizada de la educación mediática en lberoamérica se basa casi exclusivamente en los saberes, en la comprensión, en los conocimientos y no atiende las actitudes o todo aquello relacionado con lo afectivo. La educación mediática alcanzaría su esplendor si se vinculara con el conocimiento de los mecanismos mediante los que funciona el cerebro emocional. Una estrategia educativa innovadora creemos que requiere de la comprensión de las mentes de las personas que interaccionan para ser efectiva. "Si queremos formar ciudadanos autónomos y comprometidos, hay que lograr su implicación personal a través de un cambio de actitudes, no solo a través de la acumulación de conocimientos" (Figueras-Maz, Masanet y Ferrés, 2017, p. 142).

Pero el verdadero reto que compartimos los países iberoamericanos es la capacidad de integrar y valorar el conocimiento y la cultura de los estudiantes como ciudadanos, consumidores y productores audiovisuales explotando sus intereses y potenciales en clase, aprendiendo de ellos. El estudiante como centro del proceso de aprendizaje.

En esta línea, el aprendizaje colaborativo resulta clave; para García-Valcárcel, Basilotta y López Salamanca (2014), es imprescindible la transformación de las prácticas educativas a través del desarrollo de proyectos colaborativos en los que las TIC sean protagonistas. Es necesario garantizar unos escenarios de aprendizaje abiertos, interactivos, ricos en estímulos y fuentes de información, motivadores para el alumnado, centrados en el desarrollo de competencias y adaptados a los diferentes intereses y capacidades.

La educación informal es también clave. Las TIC han abierto un espacio de intercambio que fomenta la cultura participativa y se convierten en potentes entornos educativos, en donde la curiosidad, el interés y la motivación son esenciales para construir un aprendizaje colectivo basado en compartir todo aquello que se aprende con el consecuente sentimiento de solidaridad y comunidad que ello genera. Tal como afirma Henry Jenkins (2012) en una entrevista publicada en el Portal de Comunicación de la Universidad de Alcalá, en estos contextos mediados por las plataformas digitales "aprender no es el objetivo explícito de la actividad; aprender es un efecto colateral de la producción creativa, de la colaboración y la organización comunitaria" (Jenkins, el gurú de la Cultura Participativa, en la UAH, 2012). En relación con el aprendizaje informal motivado por las nuevas tecnologías, Jenkins asegura que los jóvenes difícilmente hubieran aprendido tanto dentro de un aula formal buscando sacar una buena nota.

En esta línea, y respecto al enfoque exclusivo en formar consumidores críticos de los medios, se pone de manifiesto la necesidad de comprender cómo circula la información 
y no solo tener destrezas para transmitir las ideas en diferentes formatos. Eso significa ir más allá de lo que tradicionalmente se entiende como alfabetización en los medios, enfocada al consumo crítico. Tal como reflexiona Jenkins en la misma entrevista, "una persona no está alfabetizada si solo sabe leer, pero no escribe" (Jenkins, 2012, p. 2). De la misma manera, tampoco se puede considerar que una persona está alfabetizada desde el punto de vista de los medios si sabe consumir críticamente pero no sabe producir ética ni responsablemente. Por ello, otro de los retos del sistema educativo es formar a los jóvenes para que sean usuarios creativos, eficaces y responsables de las tecnologías de la información y de la comunicación.

Es necesario que desde la escuela se reconozcan las posibilidades asociadas al uso de las TIC, no tanto desde el punto de vista técnico, sino en relación con nuevas formas de expresión que generan estos medios. Henry Jenkins en esa entrevista afirma que "las escuelas necesitan preparar a los estudiantes... para construir conocimientos individuales y también resolver problemas complejos que requieran esfuerzos colectivos... han de enseñar la ética que implica trabajar en este contexto..." (Jenkins, el gurú de la Cultura Participativa, en la UAH, 2012). Para ello los educadores muy posiblemente tendrán que familiarizarse con los nuevos medios y aprender de ellos tal como lo han hecho sus estudiantes, participando activa y colaborativamente para solo así entender las maneras de ser y hacer de este nuevo contexto.

La verdadera transformación se dará cuando los profesores reconozcan el potencial y conocimiento de sus alumnos en este contexto y recurran a ellos en busca de su ayuda para lograr su propia inmersión en las nuevas formas de comunicar, aprender y enseñar. Es entonces, cuando cambien los roles de estudiantes y docentes, que el aprendizaje será mutuo.

\section{DISCUSIÓN}

Tal como afirma Francesc Pedró en el informe Tecnologías para la Transformación de la Educación, de la Unesco y Fundación Santillana (Pedró, 2017), podemos decir que existen cambios de primer y segundo orden, y que la categorización en uno u otro grupo depende de su nivel de profundidad. Por ejemplo, se pueden entender como cambios de primer orden aquellos en los que las herramientas tecnológicas no implican un cambio estructural, es decir, continúan aplicando el modelo de educación existente, pero con el soporte de las TIC. Un ejemplo de ello sería utilizar los procesadores de texto para elaborar resúmenes u otros documentos. Los de segundo orden sí que constituyen un cambio de enfoque y procedimientos del modelo educativo, una transformación integral basada en el uso de las TIC, como por ejemplo el uso de recursos transmedia para el trabajo en relación con la producción y el consumo crítico (selección de fuentes, derechos de autoría, entre otros). A la vista de lo analizado, nos encontramos todavía en un momento 
de cambios de primer orden en el conjunto de países iberoamericanos, que puntualmente pueden coexistir con iniciativas de centros educativos, docentes individuales o experiencias concretas que se podrían categorizar como cambios de segundo orden.

Todo lo expuesto en este artículo reafirma la idea de que, más allá de la frecuencia de uso de las TIC, lo verdaderamente importante es el empleo que se les da, el cómo y el para qué de estas herramientas. En suma, podríamos afirmar que la calidad educativa no tiene relación directa con la utilización de las TIC en el aula con propósitos meramente instrumentales, sino como herramientas tecnológicas integradas a un proyecto global, flexible y con capacidad de adaptarse a las nuevas formas de entender el mundo. Por eso, integrar en los currículos escolares una nueva forma de aprender, no solo en lo técnico sino, sobre todo, en lo conceptual, supondría pasar de las iniciativas de éxito docente individuales a una transformación global e integral. La educación mediática a través de las TIC debe ser parte sustancial de las políticas públicas en materia de educación.

Solo con un cambio en los paradigmas tradicionales y el entendimiento de la tecnología como una herramienta transformadora de procesos educativos se podrán lograr mejores resultados y cambiar la práctica habitual basada en utilizar la tecnología de manera instrumental. Todo ello nos llevaría a entender la necesidad de la educación mediática con y para los docentes; sin embargo, en la mayoría de los países iberoamericanos, las políticas públicas en educación mediática muchas veces se han reducido a formación técnica del profesorado y, por tanto, al igual que con la incorporación de tecnologías en el aula, no han funcionado, puesto que no comportan un cambio de paradigma.

En el caso de España se ha puesto de manifiesto no solo la falta de asignaturas centradas en la educación mediática en el sistema universitario y sus carencias (Ferrés y Masanet, 2015), sino también la necesidad de que en estas materias se apliquen nuevos mecanismos de enseñanza/aprendizaje basados en la innovación (Fueyo, RodríguezHoyos y Linares, 2015). La reivindicación de una asignatura sobre educación mediática sigue siendo necesaria. La simple mención como eje transversal en los contenidos curriculares no es suficiente. En una situación de cambio de segundo orden sería una buena opción, pero en la fase inicial en la que nos encontramos son necesarias asignaturas específicas en los planes de estudio de todos los niveles educativos.

Tal como afirman Figueras-Maz, Masanet y Ferrés, "la integración de los dispositivos móviles en el aula será innovadora y eficaz si saca partido de las mejores prestaciones de las nuevas tecnologías y de las nuevas prácticas comunicativas: la creatividad y la participación, el trabajo en equipo, el aprendizaje permanente y la colaboración abierta y en red, la comunicación multimedial y multimodal, la interacción, la búsqueda de la implicación y de la motivación" (2017, p. 139).

Gracias a las nuevas tecnologías y formas de comunicar, los jóvenes hoy han dejado de ser meros consumidores para convertirse en prosumidores. Tal como señala Scolari, 
“las nuevas formas de narración digital, los videojuegos masivos en línea y la producción de machinima a cargo de los usuarios o el uso de plataformas como YouTube para difundir, comentar y redistribuir videos son solo algunos de los rasgos emergentes de esta nueva cultura colaborativa" (Scolari, 2016, p. 4).

En esta nueva realidad, se evidencia la necesidad de generar un aprendizaje colectivo, en el que todos comparten lo que saben con la comunidad; emerge, además, el sentido crítico, la capacidad creativa y el respeto a la diversidad, como ejes de la generación de conocimiento colectivo. El resultado deberá ser una nueva ética de la comunicación. En este contexto el sistema educativo tiene el reto de potenciar la cultura participativa y romper, o por lo menos diluir, las barreras entre el aula y la vida cotidiana de los jóvenes, sacando partido a sus motivaciones y destrezas, e integrando sus vivencias personales y colectivas, la razón con la emoción y el aprendizaje con el ocio.

Así, tras todo lo expuesto y como resultado de la revisión de literatura y el análisis de los resultados parciales de la investigación, se presenta el reto de trabajar para que las experiencias innovadoras con dispositivos móviles en el sistema educativo iberoamericano dejen de ser limitadas y puntuales. Para ello, los resultados de la investigación apuntan a la necesidad de formar a los docentes no solo en lo instruccional sino en cuanto a metodologías innovadoras y trabajar en conjunto con los responsables de innovación docente en el diseño de políticas públicas.

De este modo se reafirma que, si bien las herramientas TIC son una oportunidad para innovar y crear espacios colaborativos de trabajo, su uso sigue siendo, en términos generales, meramente instruccional. Por ello, resulta indispensable un cambio de paradigma que involucre y motive primero a docentes y responsables de innovación, para que ellos, con una visión centrada en las diversas competencias del alumnado, sean capaces de trasladar esta motivación a los estudiantes y explorar conjuntamente las posibilidades que pueden ofrecer las TIC dentro y fuera del aula.

\section{REFERENCIAS}

Andrada, P. (2018). La educación mediática en la formación de profesionales de la educación infantil en Chile. Evaluación de planes de estudio, creencias y percepción de la competencia mediática. Tesis doctoral, Barcelona, Universitat Pompeu Fabra (inédita).

Barón, L., Acevedo, F. y Luque, G. (2002). Internet, guerra y paz en Colombia. Bogotá: Antropos.

Blïnk Learning (2018). IV Estudio sobre el uso de la tecnología en la educación: España, Colombia, México, Perú y Chile. Blïnk Learning, (6), 34-52. Recuperado 
de https://www.realinfluencers.es/2018/11/13/iv-estudio-sobre-el-uso-de-la -tecnologia-en-la-educacion/

Cuban, L. (2003). Oversold and Underused. Computers in the Classroom. Cambridge: Harvard University Press

Ferrés, J., Aguaded-Gómez, I. y García-Matilla, A. (2012). La competencia mediática de la ciudadanía española: dificultades y retos. Revista ICONO14 Revista Científica de Comunicación y Tecnologías Emergentes, 10(3), 23-42. https://doi.org/10.7195/ ri14.v10i3.201

Ferrés, J. y Masanet, M.J. (2015). La educación mediática en la universidad española. Barcelona: Gedisa, S.A.

Ferrés, J. y Piscitelli, A. (2012). Media competence. Articulated proposal of dimensions and indicators. Comunicar, 19(3), 75-82. https://doi.org/10.3916/C38-2012-02-08.

Figueras-Maz, M., Masanet, M.J. y Ferrés, J. (2017). Mobile devices in higher education: A pending issue in multidimensional media literacy. Catalan Journal of Communication \& Cultural Studies, 1(9), 125-144, https://doi.org/10.1386/ cjcs.9.1.135_1

Frau-Meigs, D. (2012). Transliteracy as the new research horizon for media and information literacy. Media Studies, 3(6), 14-26. Recuperado de https://hrcak. srce.hr/index.php?id_clanak_jezik=141821\&show=clanak

Fueyo, A., Rodríguez-Hoyos, C. y Linares, C. (2015). La formación en educación mediática de los profesionales de la educación. En Fueyo, A., Rodríguez-Hoyos, C. y Tornero, J. M. (Editores). Los territorios de la Educación Mediática: experiencias en contextos educativos. Barcelona: UOC Press.

Fundación Telefónica Vivo (2016). Experiencias Evaluativas de Tecnologías Digitales en la Educación. Sao Paulo. Recuperado de http://disde.minedu.gob.pe/ bitstream/handle/MINEDU/5288/Experiencias $\% 20$ evaluativas $\% 20 \mathrm{de} \% 20$ tecnolog\%C3\%ADas\%20digitales\%20 en 20 la\%20 educaci\%C3\%B3n. pdf?sequence $=1 \&$ is Allowed $=y$

García-Valcárcel, A., Basilotta, V. y López Salamanca, C. (2014). Las TIC en el aprendizaje colaborativo en el aula de primaria y secundaria. Comunicar, 42, 65-74.http:// dx.doi.org/10.3916/C42-2014-06

Hernández, H. y Bautista, S. (2017). Las TIC en el sistema educativo mexicano. Revista Electrónica sobre Tecnología, Educación y Sociedad, 4(7), 1-13. Recuperado de http://www.ctes.org.mx/index.php/ctes/article/view/656 
Jenkins, el gurú de la Cultura Participativa, en la UAH. (23 de mayo del 2012). Portal de Comunicación de la Universidad de Alcalá. Recuperado de http:// portalcomunicacion.uah.es/diario-digital/archivo/24-archivo-entrevistasreportajes/6181-jenkins-el-guru-de-la-cultura-participativa-en-la-uah.html

Lévy, P. (2004). Inteligencia colectiva. Por una antropología del ciberespacio. Washington. Recuperado dehttp://inteligenciacolectiva.bvsalud.org/public/docu ments/pdf/es/inteligenciaColectiva.pdf

López, L. y Aguaded, M. C. (2015). Teaching media literacy in colleges of education and communication, Comunicar, 22(44), 187-95. https://doi.org/ 10.3916/ C44-2015-20.

Lugo, M.T. (2010). Las políticas TIC en la educación de América Latina. Tendencias y experiencias. Revista Fuentes, 10, 52-68.

Marta-Lazo, C., Grandío, M. M. y Gabelas, J. A. (2014). La educación mediática en las titulaciones de Educación y Comunicación de las universidades españolas. Análisis de los recursos recomendados en las guías docentes. Vivat Academia, 126, 63-78. Recuperado de http://www.vivatacademia.net/index.php/vivat/ article/view/554

Martín-Barbero, J. y Téllez, P. (2006). Los estudios de recepción y consumo en Colombia. Diálogos de la Comunicación, 73, 57-69. Recuperado de http://goo.gl/J1Xa5e

Masanet, M. J., Contreras, P. y Ferrés, J. (2013), High qualified students? Research into the media competence level of Spanish youth, Communication and Society, 26(4), 217-34. Recuperado de http://dadun.unav.edu/bitstream/10171 /35569/1/20131028165913.pdf

Morduchowicz, R. (2017). Media Literacy in Latin America. En De Abreu, B. International Handbook of Media Literacy Education. New York: Routledge. Recuperado de https://www.routledgehandbooks.com/doi/10.4324/9781315628110.ch3

OCDE (2012). Resultados de PISA 2012 en foco. Recuperado de https://www.oecd.org/ pisa/keyfindings/PISA2012_Overview_ESP-FINAL.pdf

OCDE (2015). Students, Computers and Learning: Making the Connection. Recuperado de http://www.oecd.org/publications/students-computers-and-learning-97892 64239555-en.htm

Organización de Estados Iberoamericanos para la Educación, la Ciencia y la Cultura (OEI) y Fundación Telefónica (2018). Estudio sobre la inclusión de las TIC en los centros educativos de aulas de Fundación Telefónica. Recuperado de: https://www.fundaciontelefonica.com/arte_cultura/publicaciones-listado/ pagina-item-publicaciones/itempubli/635/ 
Organización de las Naciones Unidas para la Educación, la Ciencia y la Cultura [Unesco] (2014). Enfoques estratégicos sobre las TIC en educación en América Latina y el Caribe. Recuperado de https://unesdoc.unesco.org/ark:/48223/pf0000223251

Pedró, F. (2017). Tecnologías para la transformación de la educación. España: Fundación Santillana. Recuperado de http://www.fundacionsantillana.com/PDFs/ Tecnologias\%20para\%20la\%20transformacion\%20de\%20la\%20educacion.pdf

Pegurer, M. y Martínez-Cerdá, J.F. (2016). Media Literacy in Brazil: Experiences and Models in Non-Formal Education. Comunicar, 24(49), 39-48. http://dx.doi. org/10.3916/C49-2016-04

Prensky, M. (2001) Digital natives, Digital Immigrants. On the Horizon 9(5), 1-6. Recuperado de https://marcprensky.com/writing/Prensky\%20-\%20Digital\%20Natives,\%20 Digital\%20Immigrants\%20-\%20Part1.pdf

Sandoval-Romero, Y. y Arenas, H. (2010). Mirando cómo miramos: una propuesta desde la comunicación y la educación para multiplicar miradas. Cali: USC.

Scolari, C. A. (2016). Alfabetismos transmedia. Estrategias de aprendizaje informal y competencias mediáticas en la nueva ecología de la comunicación. Telos, 193, 13-23. Recuperado de https://repositori.upf.edu/ handle/10230/27788?locale-attribute=es

Tucho, F., Fernández-Planells, A., Lozano, M. y Figueras-Maz, M. (2015). La educación mediática, una asignatura pendiente en la formación de periodistas, publicitarios y comunicadores audiovisuales. Revista Latina de Comunicación Social, 70, 689-702, https://doi.org/10.4185/RLCS-2015-1066.

Vega, J. y Lafaurie, A. (2013). A Children's Observatory of Television: "Observar TV", a Space for Dialogue between Children. Comunicar, 40, 145-153http://dx.doi. org/10.3916/C40-2013-03-05 
